\title{
Can space-based technologies help manage and prevent pandemics?
}

To the Editor-The COVID-19 pandemic has put the world at a standstill and has changed the way everyone works, communicates, studies and provides healthcare. Global vaccination strategies offer hope, but many health inequalities hinder access to vaccines, and there are increasing concerns about SARS-CoV-2 variants.

All professions are exploring innovative approaches to mitigate the challenges of COVID-19. Space stakeholders have long recognized their potential to help population health. The United Nations Conference on the Exploration and Peaceful Uses of Outer Space held in 1999 made a commitment to use space technology to help control infectious diseases. Space technologies are already assisting several aspects of public health and global health, including monitoring the environment and climate change, and assisting disaster relief ${ }^{1-5}$. Space also helped tackle the Ebola outbreak by using satellite imagery to plot disease transmission and plan relief missions, as well as by developing diagnostic labs used to test medications against Ebola ${ }^{1,2,6}$.

Space assets are now supporting COVID-19 pandemic efforts but have the potential to serve an even greater role in enhancing the monitoring and mitigation of COVID-19, and in the preparedness and prevention of future pandemics. Potential areas include tele-epidemiology, virtual care, space spinoffs, supporting One Health approaches and mental health.

Earth Observation Epidemiology or tele-epidemiology is defined as 'using space technology with remote sensing in epidemiology ${ }^{\prime, 2,4}$. It is a useful tool that is increasingly being used by clinicians and stakeholders for zoonotic infections $s^{1,2,4}$. Tele-epidemiology helped map out the spread of the Ebola virus among animals and can be used for risk mapping, risk communication and identifying vulnerable populations. Similarly, geographic information science technology can improve the understanding and control of COVID-19 through surveillance, data sharing, digital contact tracing and investigation of risk factors and infectious disease forecasting 7 . In June 2020, the US National Aeronautics and Space Administration (NASA), the European Space Agency and the Japan Aerospace

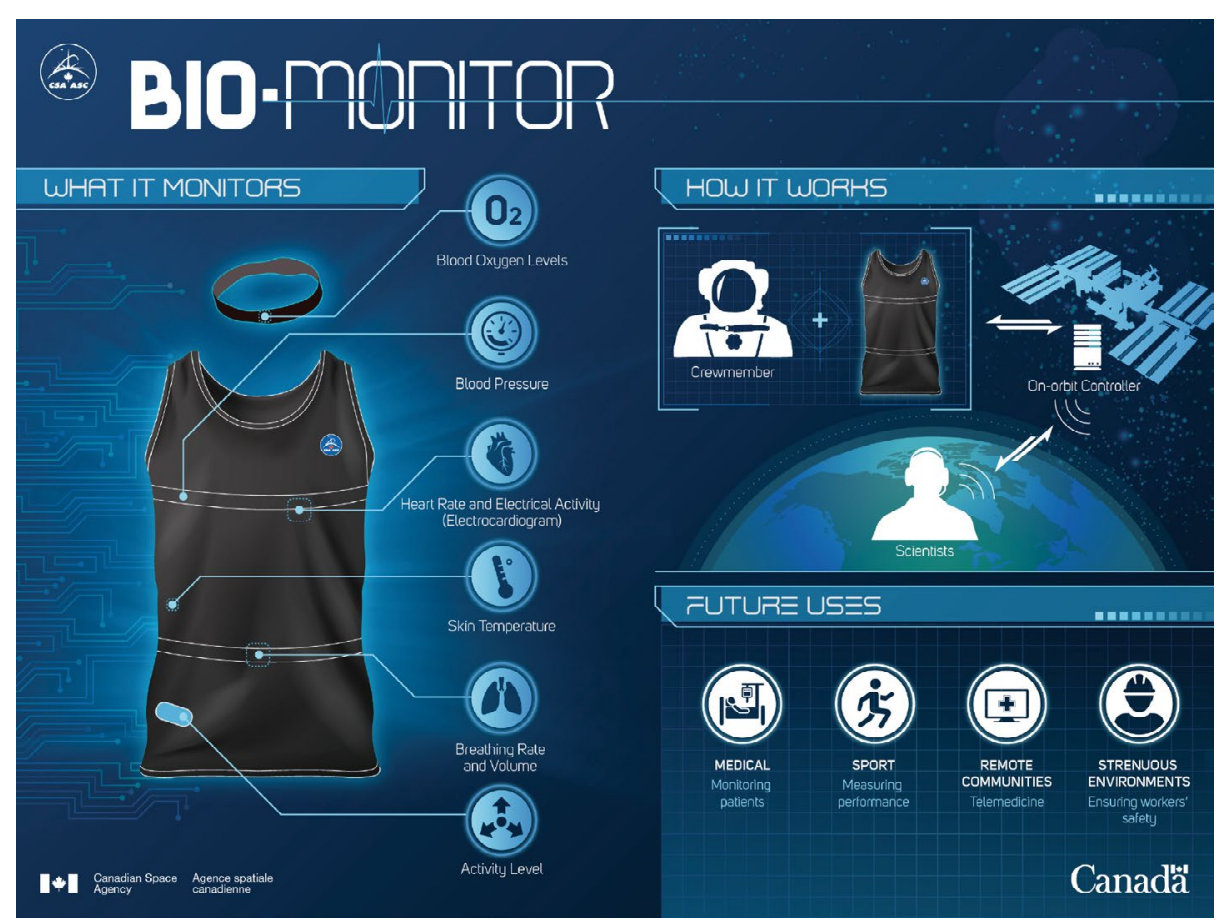

Fig. 1 | The BIO-MONITOR developed for the Canadian Space Agency. The BIO-MONITOR offers continuous monitoring of heart rate, breathing, blood pressure, temperature, electrocardiogram, physical activity and blood oxygen levels. Credit: Canadian Space Agency.

Exploration Agency joined forces to develop the COVID-19 Earth Observation Dashboard (https://eodashboard.org/), which documents planet-wide changes due to the COVID-19 pandemic.

Satellite imagery, geographic information science and global positioning system technology can also contribute to pandemic planning and the distribution of vaccines against COVID-19, particularly in lowand middle-income countries. Bill Gates noted that satellite images and global positioning system technology-enabled phone technologies were crucial in the fight against polio. Satellite images found marginalized villages in Nigeria unknown to the government, and this helped in the delivery of vaccines against polio ${ }^{8}$.

Telemedicine has served an essential role in the mitigation of COVID-19 in providing medical care and exploring options for the remote monitoring of patients with active COVID-19 or long COVID. As the space sector has been in the forefront in the development of telemedicine to provide care to and ongoing monitoring of astronauts, satellite-based communication can enhance telemedicine and expand healthcare to rural and remote areas ${ }^{1,2,4-6}$.

Space spinoffs involve making use of technology on Earth that was originally developed for space and/or by space agencies, and these are increasingly serving an important part in healthcare on Earth. The space sector has developed specialized portable clinical devices that offer continuous real-time monitoring. The BIO-MONITOR was developed for the Canadian Space Agency to store and forward physiologic data from unobtrusive body-worn sensors (Fig. 1). This wearable technology offers around-the-clock monitoring of heart rate, breathing, blood pressure, temperature, electrocardiogram, physical activity and blood oxygen levels, which are valuable parameters to monitor in patients with active COVID-19 or long COVID. Such devices could also be used to monitor the health of healthcare personnel, who are tirelessly working through the 
pandemic. Other space-based technology that are being used during the COVID-19 pandemic include point-of-care ultrasound and the European Space Agency's Biological Light Fieldable Laboratory for Emergencies to boost biomedical capabilities ${ }^{6}$. The latter is a lightweight, mobile, autonomous laboratory that can be deployed in emergencies.

An International Space University report exploring space's assets during the COVID-19 pandemic recommended using space-enabled unmanned aerial vehicles to assist with pandemic management ${ }^{2}$. A joint initiative with the UK Space Agency and European Space Agency involves a space company, Skyports, that utilizes drones using satellite communications and Earth-observing satellite data to deliver medical supplies ${ }^{2}$.

With three fourths of emerging infectious diseases being of zoonotic origin, understanding the dynamics of vector and disease spread in human and animal populations can be accomplished only by a One Health approach that leverages multiple disciplines. Remote sensing data provide real-time information about the dynamic processes of the surrounding ecosystem and air-pollution trends, as well as helping track and forecast vector-borne disease outbreaks ${ }^{1-5}$. During the COVID-19 pandemic, NASA has contributed research expertise and solicitations for the use of satellite data. Moreover, the Group on Earth Observations and the United Nations Office for Outer Space Affairs coordinated and connected global scientific networks working on integrating satellite data with ground-based public-health surveillance records.

The pandemic's increasing death toll, loss of loved ones, social-distancing restrictions and limitations to healthcare access have had a substantial impact on mental health ${ }^{2}$. The space sector has considerable experience managing astronaut self-isolation and confinement to small spaces for long periods, and thus can contribute substantially to global dialog on managing stressors and self-isolation during pandemics. NASA previously supported Earth-based emergencies, such as providing support to the 2010 efforts to rescue 33 Chilean miners trapped and isolated underground ${ }^{4,5}$. Similarly, NASA has provided numerous resources and support for managing the effects of isolation during the COVID-19 pandemic ${ }^{2}$.

Space agencies from around the world signed the International Charter on Space and Major Disasters, which provides free satellite images to support disaster-relief efforts $^{1,2,4}$. This charter provides satellite images to various organizations and governments (e.g., the United Nations, the Red Cross and Médecins Sans Frontières) to assess the impact of an emergency and to plan relief efforts ${ }^{1,2,4,5}$. The International Space University report recommended an approach similar to that of the charter, but specifically for pandemics, either through the creation of a separate charter or through modification of the existing charter to cater to the specific needs of pandemics ${ }^{2}$.

It will undoubtedly be helpful to introduce mechanisms to improve access to space technologies by relevant stakeholders, as this would provide satellite images to assist various aspects of the pandemic, such as physical-distancing measures, and to support medicine and supply-chain issues. Such images would also be useful for pandemic and vaccine planning, such as identifying strategic locations for coordinating vaccine clinics and distribution areas. Better access to space technologies would also serve a crucial role in the continued monitoring and prevention of pandemics through the use of satellite data for modeling infection transmission and assessing various pandemic risk factors, including human and vector activities, and the various roles of the environment, climate and ecology.

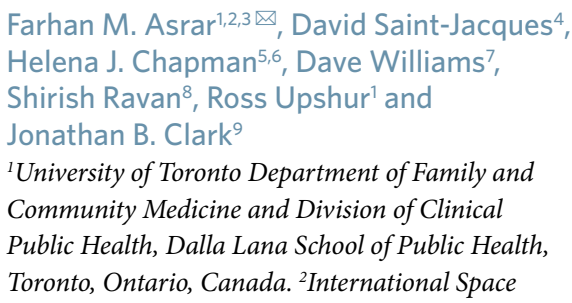

University, Strasbourg, France. ${ }^{3}$ Credit Valley Hospital and Mississauga Hospital Family Medicine Teaching Units, and the Institute for Better Health, Trillium Health Partners, Mississauga, Ontario, Canada. ${ }^{4}$ Canadian Space Agency, John H. Chapman Space Centre, Longueuil, Quebec, Canada. ${ }^{5}$ Earth Science Division, National Aeronautics and Space Administration, Washington, DC, USA. ${ }^{6}$ Booz Allen Hamilton, McLean, VA, USA. ${ }^{7}$ LEAP Biosystems, Halifax, Nova Scotia, Canada. ${ }^{8}$ United Nations Office for Outer Space Affairs, Vienna, Austria. ${ }^{9}$ Department of Neurology/Center for Space Medicine, Baylor College of Medicine, Houston, TX, USA.

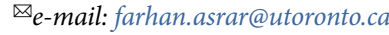

Published online: 13 September 2021 https://doi.org/10.1038/s41591-021-01485-5

References

1. Asrar, F. M. et al. Lancet Infect. Dis. 15, 873-875 (2015).

2. International Space University. https://isulibrary.isunet.edu/index. php?lvl=notice display\&id $=11027$ (2020).

3. Asrar, F. M. et al. Lancet Planet Health 2, e469-e470 (2018).

4. International Space University. https://isulibrary.isunet.edu/index. php?lvl=notice_display\&id=9175 (2014).

5. Dinas, P. C. et al. Disaster Med. Public Health Prep. 9 , 319-328 (2015)

6. Holmes, M. Via Satellite http://interactive.satellitetoday.com/via/ august-2020/satellites-enhanced-role-in-space-based-healthcare/ (2020).

7. Smith, C. D. \& Mennis, J. Prev. Chronic Dis. 17, E58 (2020).

8. Gates, B. GatesNotes https://www.gatesnotes.com/health/ gis-mapping-gps-tracking-for-polio-in-nigeria (2012).

\section{Acknowledgements}

We acknowledge A. G. Karacalığlu and J. de Dalmau of the International Space University, as well as the faculty, staff and participants of the inaugural International Space University Interactive Space Program (ISP20). F.M.A. was invited by the International Space University to chair an international team involving over 100 professionals, faculty members and experts from over 30 countries that researched the role of space during pandemics (20202021), who presented their findings to the United Nations Office for Outer Space Affairs. J.B.C. was a six-time space shuttle crew surgeon at NASA from 1997 to 2005. D.S.J. is a current astronaut who trialled the BIO-MONITOR aboard the International Space Station, and D.W. is a former astronaut with the Canadian Space Agency.

\section{Author contributions}

All authors contributed to the content of the manuscript, providing expertise from their respective areas and editing and revising the manuscript. F.M.A. came up with the concept and idea for the manuscript and wrote the first draft.

Competing interests

The authors declare no competing interests. 